\title{
COASSOCIATED PRIMES OF MODULES OVER A COMMUTATIVE RING
}

\author{
SIAMAK YASSEMI
}

\begin{abstract}
.
In this paper the concept of the coassociated prime of modules over (not necessarily Noetherian) commutative rings is introduced.
\end{abstract}

\section{Introduction.}

The theory of associated primes is very important in the study of modules over commutative Noetherian rings. For modules over non-Noetherian rings however, the classical associated primes do not behave so well, for example there exist non-trivial modules without associated primes. In [1] Bourbaki has introduced the notion of weakly associated primes of $M$ over a (not necessarily Noetherian) commutative ring $R$ : the prime ideal $p$ is weakly associated to $M$ if there exists an element $x \in M$ such that $p$ is a minimal among the prime ideals contaning the annihilator Ann $x$. In this paper the set of weakly associated primes of $M$ is denoted by A $\widetilde{s s} M$. In [2] Iroz and Rush have studied further the associated primes of modules over a (not necessarily Noetherian) commutative ring $R$. They have shown that the set A $\widetilde{s S} M$ of weakly associated primes of $R$-module $M$ is -- in some sense -- the best choice for a notion of associated primes over a (not necessarily Noetherian) commutative ring.

The aim of this paper is to develop a theory dual to that of weakly associated primes.

Let $\mathrm{D}_{m}(-)$ denote the functor $\operatorname{Hom}(-, \mathrm{E}(R / m))$ for $m \in \operatorname{Max} R$, the maximal spectrum of $R$, and the injective envelope $\mathrm{E}(R / m)$ of $R / m$.

In [10] we have introduced the notion of a cocyclic module (that is, a submodule of $\mathrm{D}_{m}(R)$ for some $m \in \operatorname{Max} R$ ) and it is used to define the notion of coassociated primes of modules over Noetherian rings (and this is dual to the classical associated primes). In this paper we use the notion of cocyclic modules to introduce the notion of weakly coassociated prime of modules 
(dual notion to that of weakly associated prime). We define the set Coass $M$ of weakly coassociated primes of an $R$-module $M$ to be the set of prime ideals $p$ such that there exists a cocyclic homomorphic image $L$ of $M$ with $p$ is a minimal element in $\mathrm{V}(\operatorname{Ann} L)$.

We show that $p \in \widetilde{\text { Coass }} M$ if and only if there exists a maximal ideal $m$ such that $p \in \widetilde{A s s} \mathrm{D}_{m}(M)$, and this result will play an important rôle in the rest of the paper.

Also we show that if $p \in A \widetilde{s s} M$, then $p \in \widetilde{\text { Coass }} \mathrm{D}_{m}(M)$ for all ideal $m \in \operatorname{Max} R \cap \mathrm{V}(p)$ (but the inverse is not true).

It turns out that weakly coassociated primes have properties similar -- or rather dual -- to those of weakly associated primes. For example, C $\widetilde{\text { oass }} M \neq$ $\varnothing$ whenever $M$ is non-trivial, and that the set of coassociated primes of $M$ is a subset of the set of weakly coassociated primes of $M$. In addition, Coass $M=$ Coass $M$ when $R$ is Noetherian. Finally, if $0 \rightarrow M^{\prime} \rightarrow$ $M \rightarrow M^{\prime \prime} \rightarrow 0$ is an exact sequence of $R$-modules and $R$-homomorphisms, then it turns out that there are inclusions:

\section{$\widetilde{\text { Coass }} M^{\prime \prime} \subseteq$ Coass $M \subseteq$ Coass $M^{\prime} \cup \widetilde{\text { Coass }} M^{\prime \prime}$.}

In [3] Macdonald defined the set of attached primes of an $R$-module $M$. The theory of attached primes is particularly well-behaved when $M$ has a secondary representation (which is the dual notion to primary decomposition). However, in general this theory is not completely satisfactory. In this paper we prove that the set of weakly coassociated primes of $M$ is equal to the set of attached primes of $M$ when $M$ has a secondary representation.

Assume that $R$ is a Noetherian ring and the zero submodule of $M$ has primary decomposition. We find the set of coassociated primes of $\operatorname{Hom}(M, E)$ for any injective module $E$. This result is a sharpening of $[8,2.6]$ and $[9,2.1]$.

We define the cosupport of module $M$ over commutative rings (this definition is the same as definition of the cosupport over Noetherian rings, cf. [10]). It follows that the set of weakly coassociated primes is a subset of the cosupport. On the other hand every minimal element of the cosupport belongs to the set of weakly coassociated primes.

In the last section we bring some functorial results. Let $\mathscr{P}$ be the class of all modules $M$ such that the zero submodule has a primary decomposition in $M$ (or $M=0$ ), and let $\mathscr{S}$ be the class of all modules having secondary representation (or being zero). If $M \in \mathscr{P}$ then $\operatorname{Hom}(L, M) \in \mathscr{P}$ for any $R$ module $L, M \otimes F \in \mathscr{P}$ for any flat module $F$, and $\operatorname{Hom}(M, E) \in \mathscr{S}$ for any injective module $E$. If $N \in \mathscr{S}$ then $N \otimes L \in \mathscr{S}, \operatorname{Hom}(N, L) \in \mathscr{P}$ for any $R$ module $L$, and $\operatorname{Hom}(P, N) \in \mathscr{S}$ for any projective module $P$.

Throughout this paper the ring $R$ is commutative with a non-zero identity 
element. We write "finite" for "finitely generated". Also, we shall use Max $R$ to denote the set of all maximal ideals of $R$. For an $R$-module $M$ its injective envelope is denoted by $\mathrm{E}(M)$.

The author would like to express his gratitude to professor H.-B. Foxby for all his support, helpfulness, and in particular for suggesting many of the topics considered in this paper.

The author would also like to thank the University of Copenhagen and in particular the Mathematics Institute for its hospitality and the facilities offered during the preparation of this paper.

\section{Weakly associated primes.}

Let $M$ be an $R$-module. The set of weakly associated prime ideals of $M$ is denoted by A $\widetilde{s s} M$ and it is the set of prime ideals $p$ such that there exists $x \in M$ with $p$ a minimal element in $\mathrm{V}(\operatorname{Ann} x)$, that is, the set of prime ideals $p$ of $R$ such that there exists a cyclic submodule $N$ of $M$ with $p$ a minimal element in $\mathrm{V}($ Ann $N)$.

1.1. Theorem. Let $M$ be an R-module. Then the following hold

(a) Ass $M \subseteq$ A $\widetilde{s s} M \subseteq \operatorname{Supp} M$.

(b) Ass $M=$ A $\widetilde{s s} M$ if $R$ is a Noetherian ring.

(c) A $\widetilde{\text { ss }} M \neq \varnothing$ if $M \neq 0$.

(d) If $0 \rightarrow M^{\prime} \rightarrow M \rightarrow M^{\prime \prime} \rightarrow 0$ is an exact sequence, then

A $\widetilde{s s} M^{\prime} \subseteq \mathrm{A} \widetilde{s s} M \subseteq A \widetilde{A s} M^{\prime} \cup \mathrm{A} \widetilde{s s} M^{\prime \prime}$.

(e) $\mathrm{z}(M)=\bigcup_{p \in \text { Ass } M} p$.

(f) If $S$ is a multiplicative closed system of $R$, then

$$
\mathrm{Ass}_{S^{-1} R} S^{-1} M=\left\{p S^{-1} R \mid p \in \mathrm{A}_{\mathrm{As}_{R}} M \text { with } p \cap S=\emptyset\right\} .
$$

Proof. See [1, page 289--290] and [2, 6.1--6.2].

1.2. Corollary Let $M$ be a Noetherian R-module. Then

$$
\mathrm{Ass}_{R} M=\operatorname{Ass}_{R} M .
$$

Proof. Set $R^{\prime}=R /$ Ann $M$, which is a Noetherian ring, and then use (1.1) (b).

It follows from (1.1) (a) that Ass $M \subseteq$ A $\widetilde{s s} M$ and equality holds when $M$ is a Noetherian by (1.2). The next example shows that equality does not hold in general.

EXAMPLE. Let $k$ be a field and consider the ring $R=k^{\mathrm{N}}$ (direct product). Set $a=k^{(\mathrm{N})}$ (direct sum) which is an ideal of $R$. Set $M=R / a$. We claim that 
Ass $M=\varnothing$. Assume that $p \in$ Ass $M$. Then $a \subseteq p=(a: r)$ for some $r \notin a$. It is easy to find two elements $s$ and $t$ of $R$ such that $s r, t r \notin a$ and $s t=0$. Since $s t r=0$ we have $s t \in p$. But $s \notin p$ and $t \notin p$ and this is a contradiction.

An $R$-module $M \neq 0$ is said to be coprimary if for each $a \in R$ the homothety $M \stackrel{a \cdot}{\longrightarrow} M$ is either injective or nilpotent. This implies that nil $M=p$ is a prime ideal of $R$ (note that nil $M=\sqrt{\operatorname{Ann} M}$ ), and $M$ is said to be $p$-coprimary.

If there exist a finite number of primary submodules $N_{i}$ (in other word $M / N_{i}$ is coprimary) for $1 \leq i \leq n$ such that $0=N_{1} \cap N_{2} \cap \cdots \cap N_{n}$, then we say that the zero submodule of $M$ has primary decomposition. In this case $M$ is isomorphic to a submodule of $M / N_{1} \oplus M / N_{2} \oplus \cdots \oplus M / N_{n}$.

1.3. Lemma. Let the zero submodule of $M$ have a primary decomposition and let $0=N_{1} \cap N_{2} \cap \cdots \cap N_{n}$ be a minimal primary decomposition of 0 where $N_{i}$ is $p_{i}$-primary submodule of $M$ for $i=1,2, \ldots, n$. Then

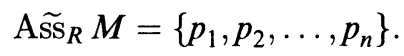

Proof. " $\subseteq$ " First assume that the zero submodule of $M$ is $p$--primary (in the other words, $M$ is $p$-coprimary). Let $q \in \mathrm{A} \widetilde{s S} M$. Then there exists a cyclic submodule $N$ of $M$ such that $q$ is a minimal element in $\mathrm{V}(\operatorname{Ann} N)$. Since $N$ is also $p$-coprimary we have nil $N=p$. Therefore $q=p$.

Now let the zero submodule of $M$ have primary decomposition. Then there exist the exact sequence $0 \rightarrow M \rightarrow \oplus\left(M / N_{i}\right)$. We have A $\widetilde{s s} M \subseteq$ A $\widetilde{s s}\left(\oplus M / N_{i}\right)=\cup \operatorname{Ass}\left(M / N_{i}\right)$ by (1.1) (d) and $\operatorname{Ass}\left(M / N_{i}\right)=\left\{p_{i}\right\}$ (as already proved). Now the assertion follows.

" $\supseteq$ " Set $K_{j}=\cap_{i \neq j} N_{i}$. Then $K_{j} \neq 0$ by the minimality of the primary decomposition, and $K_{j}=K_{j} /\left(K_{j} \cap N_{j}\right) \cong\left(K_{j}+N_{j}\right) / N_{j} \subseteq M / N_{j}$. Now choose by (1.1) (c) $q \in \operatorname{Ass} K_{j}$. Since A $\widetilde{s s} K_{j} \subseteq \operatorname{Ass}\left(M / N_{j}\right) \subseteq\left\{p_{j}\right\}$ by (1.1) (d) and the already established inclusion, we get $p_{j}=q \in \mathrm{A} \widetilde{\mathrm{ss}} K_{j} \subseteq \mathrm{A} \widetilde{\mathrm{ss}} M$ as desired.

1.4. Corollary. If the zero submodule of $M$ has a primary decomposition then $\mathrm{A} \widetilde{\mathrm{ss}} M$ is a finite set. In particular, if $M$ in a Noetherian R-module then A $\widetilde{s s} M$ is a finite set.

1.5. THEOREM. Let the zero submodule of $M$ have primary decomposition. Then the following hold.

(a) The minimal elements of the set $\mathrm{V}(\mathrm{Ann} M)$ belong to A $\widetilde{s s} M$.

(b) A $\widetilde{s s}(R / A n n M) \subseteq$ A $\widetilde{s s} M$.

(c) The sets $\mathrm{V}(\mathrm{Ann} M)$, A $\widetilde{\mathrm{ss}}(R / \mathrm{Ann} M)$ and $\mathrm{A} \widetilde{\mathrm{ss}} M$ have the same minimal elements.

Proof. Let $0=N_{1} \cap N_{2} \cap \cdots \cap N_{n}$ be a minimal primary decomposition 
of the zero submodule of $M$ where $N_{i}$ is a $p_{i}$-primary submodule of $M$ for $i=1,2, \ldots, n$.

(a) There exists an exact sequence $0 \rightarrow M \rightarrow \oplus_{i}\left(M / N_{i}\right)$. Thus $\cap_{i}$ Ann $\left(M / N_{i}\right)=$ Ann $M$ (since the inclusion $\supseteq$ is obvious). Let $p$ be a minimal element of the set $\mathrm{V}(\operatorname{Ann} M)$. Then $p$ is a minimal element of the set $\mathrm{V}\left(\operatorname{Ann}\left(M / N_{i}\right)\right)$ for some $1 \leq i \leq n$. Since $\operatorname{nil}\left(M / N_{i}\right)=p_{i}$ we have $p=p_{i}$. Now the assertion follows from (1.3).

(b) Let $p \in \operatorname{Ass}(R / A n n M)$. Then $p$ is a minimal element of the set $\mathrm{V}$ (Ann $(a+\operatorname{Ann} M))$ for some $a \in R$. Thus $p$ is a minimal element of $\mathrm{V}($ Ann $a M)$. Therefore $p \in \mathrm{A} \widetilde{\mathrm{ss}}(a M)$. Now the assersion follows from (1.1) (d).

(c) We know that $\mathrm{V}(\operatorname{Ann} M)$ and A $\widetilde{s s} M$ have the same minimal elements by (a) and the fact that $\mathrm{A} \widetilde{s S} M \subseteq \mathrm{V}(\operatorname{Ann} M)$. For any $p \in \operatorname{Ass}(R / \operatorname{Ann} M)$ we know that Ann $M \subseteq p$. Now the assertion follows from (b).

1.6. Theorem. Let $R$ be a Noetherian ring and let $F$ be a flat $R$-module. Let $p$ be a prime ideal of $R$. Then the following are equivalent

(i) There exists a p-coprimary module $M$ such that $M \otimes F \neq 0$.

(ii) For any p-coprimary module $M$ we have $M \otimes F \neq 0$.

(iii) $R / p \otimes F \neq 0$.

Proof. Let $M$ be a $p$-coprimary. Since $R$ is a Noetherian ring and $M$ has primary decomposition we have Ass $M=\{p\}$.

“(i) $\Rightarrow$ (iii)" Since Ass $M=\{p\}$ we have $\operatorname{Ass}(M \otimes F)=\operatorname{Ass}(R / p \otimes F)$ by [1, page 154]. Since $M \otimes F \neq 0$ we have $\operatorname{Ass}(M \otimes F) \neq \varnothing$ and hence $\operatorname{Ass}(R / p \otimes F) \neq \varnothing$. Now the desired implication has been established.

"(iii) $\Rightarrow$ (ii)" The exact sequence $0 \rightarrow R / p \rightarrow M$ induces the exact sequence $0 \rightarrow(R / p) \otimes F \rightarrow M \otimes F$. Now the assertion holds.

"(ii) $\Rightarrow$ (i)" This is clear.

1.7. Lemma. Let $M, N$ be $R$-modules. If $\operatorname{Hom}(M, N) \neq 0$ then there exists $p \in \operatorname{Ass} M$ such that $p \subseteq q$ for some $q \in$ A $\widetilde{s s} N$.

Proof. Let $\varphi: M \rightarrow N$ be a non-zero map. Choose $q \in \operatorname{Ass} \varphi(M)$. Then $q \in \mathrm{A} \widetilde{\mathrm{ss}} N$. Since $q \in \mathrm{Ass} \varphi(M)$, we can choose $x \in M$ such that $q$ is a minimal element in $\mathrm{V}(\operatorname{Ann}(\varphi(x)))$. Thus $q \in \mathrm{V}(\operatorname{Ann} x)$. We can choose $p$ as a minimal element of $\mathrm{V}(\operatorname{Ann} x)$ such that $p \subseteq q$. Thus $p \in \operatorname{Ass} M$.

1.8. TheOREM. Let $E$ be an injective $R$-module such that $\mathrm{A} \widetilde{\mathrm{ss}} E=\operatorname{Ass} E$. Let $p$ be a prime ideal of $R$. Then the following are equivalent

(i) There exists a p-coprimary module $M$ such that $\operatorname{Hom}(M, E) \neq 0$.

(ii) For any p-coprimary module $M$ we have $\operatorname{Hom}(M, E) \neq 0$.

(iii) $\operatorname{Hom}(R / p, E) \neq 0$. 
Proof. "(iii) $\Rightarrow$ (ii)" Since $p \in A \widetilde{A s} M$ we have $x \in M$ such that $p$ is minimal in $\mathrm{V}(\operatorname{Ann}(R x))$. The sequence $R x \rightarrow R / p \rightarrow 0$ is exact and induces the exact sequence $0 \rightarrow \operatorname{Hom}(R / p, E) \rightarrow \operatorname{Hom}(R x, E)$. Thus $\operatorname{Hom}(R x, E) \neq 0$. In addition, the exact sequence $0 \rightarrow R x \rightarrow M$ induces the exact sequence $\operatorname{Hom}(M, E) \rightarrow \operatorname{Hom}(R x, E) \rightarrow 0$. Therefore $\operatorname{Hom}(M, E) \neq 0$.

"(ii) $\Rightarrow$ (i)" This is clear.

"(i) $\Rightarrow$ (iii)" Since A $\widetilde{s s} M=\{p\}$ by (1.3), and $\operatorname{Hom}(M, E) \neq 0$ we have $p \subseteq q$ for some $q \in \operatorname{Ass} E=\operatorname{Ass} E$ by (1.7). Thus $\operatorname{Hom}(R / q, E) \neq 0$. Now the exact sequence $R / p \rightarrow R / q \rightarrow 0$ induce the exact sequence $0 \rightarrow$ $\operatorname{Hom}(R / q, E) \rightarrow \operatorname{Hom}(R / p, E)$. Thus $\operatorname{Hom}(R / p, E) \neq 0$.

1.9. Remark. If $R$ is a Noetherian ring then (i), (ii) and (iii) are equivalent for any injective $R$-module $E$.

Let $R$ be a Noetherian ring and let $N$ be a finite $R$-module. It is wellknown that for any $R$-module $M$ we have $\operatorname{Hom}(N, M) \neq 0$ if and only if $p \supseteq$ Ann $N$ for some $p \in$ Ass $M$, cf. [1, page 267]. In the next example we show that this result does not hold for A $\widetilde{s s} M$ when $R$ is not Noetherian.

Example. Let $R$ and $M$ be the same as in the example after (1.2). Assume that $m \in \mathrm{Ass} M$ so $m \in \operatorname{Max} R$ since that ring $R$ is von Neumann regular. Since $m \notin$ Ass $M$ we have $\operatorname{Hom}(R / m, M)=0$.

\section{Weakly coassociated primes.}

In this section we introduce the notion of weakly coassociated primes (dual notion to that of weakly associated prime). First we bring some definitions and results of [10].

In [10] we have introduced the notion of cocyclic module, and it is used to define the coassociated prime ideals and cosupport of modules over Noetherian rings. Now note that the next definitions are over (not necessarily Noetherian) commutative rings. For any maximal ideal $m$ of $R$ the functor $\operatorname{Hom}(-, \mathrm{E}(R / m))$ is denoted by $\mathrm{D}_{m}(-)$.

2.1. Definition. An $R$-module $L$ is said to be cocyclic if $L$ is a submodule of $\mathrm{D}_{m}(R)$ for some $m \in \operatorname{Max} R$.

2.2. Remark. The module $M$ is cocyclic if there exists an ideal $a$ of $R$ such that $M \cong \mathrm{D}_{m}(R / a)$. This follows from the fact that $\mathrm{D}_{m}(R / a) \subseteq \mathrm{D}_{m}(R)$.

2.3. Definition. Let $M$ be an $R$-module. A prime ideal $p$ of $R$ is said to be a coassociated prime of $M$ if there exists a cocyclic homomorphic image $L$ of $M$ such that $p=\operatorname{Ann} L$. The set of coassociated prime ideals of $M$ is denoted by Coass $M$. 
The next theorem was proved in $[10,1.7]$ with the Noetherian condition on $R$, but the proof in the non-Noetherian case is the same.

2.4. TheOREM. Let $M$ be an R-module. The following are equivalent

(i) $p \in$ Coass $M$

(ii) There exists $m \in \operatorname{Max} R \cap \mathrm{V}(p)$ such that $p \in \operatorname{Ass}_{m}(M)$.

2.5. Definition. For an $R$-module $M$ the subset w $(M)$ of $R$ is defined by

$$
\mathrm{w}(M)=\{a \in R \mid M \stackrel{a \cdot}{\longrightarrow} M \text { is not surjective }\} .
$$

2.6. Definition. Let $M$ be an $R$-module. The cosupport of $M$, written Cosupp $M$, is the set of prime ideals $p$ such that there exists a cocyclic homomorphic image $L$ of $M$ with $p \in \mathrm{V}(\operatorname{Ann} L)$.

2.7. Definition. Let $M$ be an $R$-module. A prime ideal $p$ of $R$ is said to be a weakly coassociated prime of $M$ if there exists a cocyclic homomorphic image $L$ of $M$ such that $p$ is a minimal element in $\mathrm{V}(\operatorname{Ann} L)$. The set of weakly coassociated prime ideals of $M$ is denoted by Coass $M$.

2.8. LemmA For any $R$-module $M$, Coass $M \subseteq$ Cosupp $M$ and every minimal element of the set $\operatorname{Cosupp} M$ belongs to Coass $M$.

Proof. It follows directly from (2.6) and (2.7).

2.9. TheOREM. Let $M$ be an $R$-module. The following are equivalent

(i) $p \in$ Coass $M$

(ii) There exists $m \in \operatorname{Max} R \cap \mathrm{V}(p)$ such that $p \in \mathrm{A}_{\tilde{s s}} \mathrm{D}_{m}(M)$.

Proof. (i) $\Rightarrow$ (ii). If $p \in \widetilde{\text { Coass }} M$, then there exists a cocyclic homomorphic image $L$ of $M$ such that $p$ is a minimal element in $\mathrm{V}(\operatorname{Ann} L)$. Let $\varphi: M \rightarrow L$ be the surjective homomorphism. Thus Ann $\varphi=\operatorname{Ann} L$. Therefore $p$ is a minimal element in $\mathrm{V}(\operatorname{Ann} \varphi)$ and hence $p \in \operatorname{Ass} \operatorname{Hom}(M, L)$. Since $L$ is a submodule of $\mathrm{D}_{m}(R)$ for some $m \in \operatorname{Max} R$, we have $p \in \operatorname{Ass} \mathrm{D}_{m}(M)$ for the same $m \in \operatorname{Max} R$ by (1.1) (d).

(ii) $\Rightarrow$ (i). If $p \in \operatorname{Ass} \mathrm{D}_{m}(M)$, then there exists $\varphi \in \mathrm{D}_{m}(M)$ such that $p$ is a minimal element in $\mathrm{V}(\operatorname{Ann} \varphi)$. Let $L=\varphi(M)$ (submodule of $\mathrm{D}_{m}(R)$ ). Thus $L$ is a cocyclic and Ann $L=\operatorname{Ann} \varphi$. Hence we have $p \in \widetilde{\operatorname{Coass}}(M)$.

2.10. TheOREM. For any R-module $M$ the following hold.

(a) Coass $M \subseteq$ Coass $M$.

(b) If $R$ is a Noetherian ring then Coass $M=$ Coass $M$.

(c) If $M$ is an Artinian $R$-module then Coass $M=\widetilde{\text { Coass }} M$. 
Proof. (a) It is clear.

(b) Let $p \in$ Coass $M$. There exists $m \in \operatorname{Max} R$ with $p \in \widetilde{\mathrm{Ass}} \mathrm{D}_{m}(M)$ and hence $p \in \operatorname{Ass} \mathrm{D}_{m}(M)$ by (1.1) (b). Therefore we have $p \in$ Coass $M$ by (2.4).

(c) Let $p \in$ Coass $M$. Then $p \in A \widetilde{A s} \mathrm{D}_{m}(M)$ for some ideal $m \in \operatorname{Max} R \cap \mathrm{V}(p)$. We have A $\widetilde{s s}\left(\mathrm{D}_{m}(M)\right)=$ Ass $\left(\mathrm{D}_{m}(M)\right)$, since $M$ is an

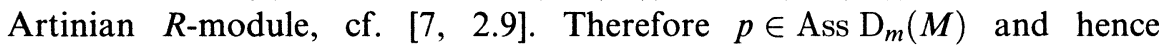
$p \in$ Coass $M$ by (2.4). Now the assertion follows from (a).

2.11. Theorem. Let $M$ be an R-module. Then Coass $M \neq \varnothing$ if $M \neq 0$.

Proof. Assume $M \neq 0$. Since $\mathrm{D}_{m}(M) \cong \operatorname{Hom}_{R_{m}}\left(M_{m}, \mathrm{E}(R / m)\right)$ we have then $\mathrm{D}_{m}(M) \neq 0$ for some $m \in \operatorname{Max} R$ (namely for $m \in \operatorname{Supp} M \cap \operatorname{Max} R$ ). Thus $\mathrm{A} \widetilde{\mathrm{ss}} \mathrm{D}_{m}(M) \neq \varnothing$. Hence Coass $M \neq \varnothing$ by (2.9).

2.12. THEOREM. If $0 \rightarrow M^{\prime} \rightarrow M \rightarrow M^{\prime \prime} \rightarrow 0$ is an exact sequence, then Coass $M^{\prime \prime} \subseteq$ Coass $M \subseteq$ Coass $M^{\prime} \cup \widetilde{\text { Coass }} M^{\prime \prime}$.

Proof. If $p \in$ Coass $M^{\prime \prime}$, then there exists $m \in \operatorname{Max} R \cap \mathrm{V}(p)$ such that $p \in \operatorname{Ass} \mathrm{D}_{m}\left(M^{\prime \prime}\right)$ by (2.9). We have an exact sequence

$$
0 \rightarrow \mathrm{D}_{m}\left(M^{\prime \prime}\right) \rightarrow \mathrm{D}_{m}(M) \rightarrow \mathrm{D}_{m}\left(M^{\prime}\right) \rightarrow 0 .
$$

Thus $p \in \widetilde{\operatorname{Ass}} \mathrm{D}_{m}(M)$. Hence $p \in$ Coass $M$ by (2.9).

If now $p \in$ Coass $M$ then there exists $m \in \operatorname{Max} R \cap \mathrm{V}(p)$ such that $p \in \operatorname{A~ss~}_{m}(M)$ by (2.9). Consider once more the exact sequence $(*)$. It follows that $p \in \widetilde{\operatorname{Ass}} \mathrm{D}_{m}\left(M^{\prime \prime}\right)$ or $p \in \widetilde{\operatorname{Ass}} \mathrm{D}_{m}\left(M^{\prime}\right)$, and hence $p \in$ Coass $M^{\prime \prime}$ or $p \in$ Coass $M^{\prime}$ by (2.9).

2.13. Corollary. For $R$-modules $M_{1}, \ldots, M_{n}$ we have

$$
\widetilde{\operatorname{Coass}}\left(M_{1} \oplus \cdots \oplus M_{n}\right)=\widetilde{\operatorname{Coass}}\left(M_{1}\right) \cup \cdots \cup \widetilde{\operatorname{Coass}}\left(M_{n}\right) \text {. }
$$

ProOF. "C" It is clear from (2.12) by induction.

" $\supseteq$ " It follows from the fact that $M_{i}$ is a homomorphic image of $M_{1} \oplus \cdots \oplus M_{n}$ for all $i$.

2.14. Lemma. Let $M$ be an $R$-module. If $p \in \mathrm{A} \widetilde{s s} M$, then $p \in \widetilde{\text { Coass }} \mathrm{D}_{m}(M)$ for all $m \in \operatorname{Max} R \cap \mathrm{V}(p)$.

Proof. Let $p \in \mathrm{A} \widetilde{s \mathrm{~s}} M$ and let $m \in \operatorname{Max} R \cap \mathrm{V}(p)$. Then there exists $x \in M$ such that $p$ is a minimal element in $\mathrm{V}(\operatorname{Ann} x)$. The exact sequence $R x \rightarrow R / p \rightarrow 0$ induced the exact sequence $0 \rightarrow \mathrm{D}_{m}(R / p) \rightarrow \mathrm{D}_{m}(R x)$. Since Ann $\left(\mathrm{D}_{m}(R / p)\right)=p$ and Ann $x \subseteq \operatorname{Ann}\left(\mathrm{D}_{m}(R x)\right)$, we have $p$ is a minimal element in $\mathrm{V}\left(\operatorname{Ann}\left(\mathrm{D}_{m}(R x)\right)\right)$. On the other hand, Since $R x \cong R / \operatorname{Ann} x$, we have that $\mathrm{D}_{m}(R x)$ is a cocyclic by (2.2). Thus $p \in \widetilde{\text { Coass }} \mathrm{D}_{m}(R x)$ and hence $p \in$ Coass $\mathrm{D}_{m}(M)$ by $(2.12)$. 
2.15. ThEOREM. For any $R$-module $M$ there is an equality

$$
\mathrm{w}(M)=\bigcup_{p \in \text { Coass } M} p .
$$

Proof. If $a \in \bigcup_{p \in \widetilde{\operatorname{Coass}} M} p$, then there exists $p \in \widetilde{\text { Coass }} M$ with $a \in p$. By

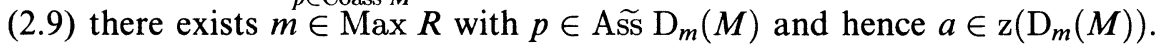
Thus $a M \neq M$ and hence $a \in \mathrm{w}(M)$.

On the other hand let $a \in(M)$. Since $M / a M \neq 0$, we have $\widetilde{\operatorname{Coass}}(M / a M) \neq \varnothing$ by (2.11). Let $p \in \widetilde{\operatorname{Coass}}(M / a M)$. Then $a \in p$ and $p \in \widetilde{\text { Coass }} M$ by (2.12). Therefore $a \in \bigcup_{p \in \widetilde{\operatorname{Coass}} M} p$.

\section{Attached primes.}

In [3] I. G. Macdonald has developed the theory of attached prime ideals and secondary representation of a module, which is (in a certain sense) a dual to the theory of associated prime ideals and primary decompositions. Now we want to prove that if $M$ has a secondary representation then $\operatorname{Att}(M)=\widetilde{\operatorname{Coass}}(M)$. We use the notation of [3]. An $R$-module $M \neq 0$ is called secondary if for each $a \in R$ multiplication by $a$ on $M$ is either surjective or nilpotent. Then nil $M=p$ is a prime ideal and $M$ is called $p$-secondary. We say that $M$ has a secondary representation if there is a finite number of secondary submodules $M_{1}, M_{2}, \ldots, M_{n}$ such that $M=M_{1}+M_{2}+\cdots+$ $M_{n}$. One may assume that the prime ideals nil $M_{i}=p_{i}, i=1,2, \ldots, n$, are all distinct and, by omitting redundant summands, that the representation is minimal. Then the set of prime ideals $\left\{p_{1}, \ldots, p_{n}\right\}$ does not depend on the representation, and it is called the set of attached prime ideals and denoted by $\operatorname{Att}(M)$. In [3] Macdonald showed that Artinian modules have secondary representation.

3.1. THEOREM. If $M$ have a secondary representation and $M=S_{1}+$ $S_{2}+\cdots+S_{n}$ is a minimal secondary representation of $M$ where $S_{i}$ is a $p_{i}$-secondary submodule of $M$ for $i=1,2, \ldots, n$, then

$$
\widetilde{\mathrm{Coass}} M=\left\{p_{1}, p_{2}, \ldots, p_{n}\right\}=\operatorname{Att} M \text {. }
$$

Proof. First assume that $M$ is a $p$-secondary. Then Att $M=\{p\}$. Let $q \in$ Coass $M$. There exists then a cocyclic homomorphic image $L$ of $M$ such that $q$ is a minimal element in $\mathrm{V}(\operatorname{Ann} L)$. Since $L$ is also $p$-secondary we have nil $L=p$ and hence $q=p$.

Now let $M$ have secondary representation. Then there exists an exact sequence $\oplus S_{i} \rightarrow M \rightarrow 0$. Since Coass $M \subseteq \cup_{i}$ Coass $S_{i}$ by (2.12) and (2.13), we have Coass $M \subseteq$ Att $M$ (since it has already been proved that $\widetilde{\text { Coass }} S_{i}=$ Att $S_{i}$ for any $\left.i=1,2, \ldots, n\right)$. 
Set $K_{j}=\sum_{i \neq j} N_{i}$. Then $M / K_{j} \neq 0$ by the minimality of the representation, and $M / K_{j}=\left(K_{j}+S_{j}\right) / K_{j} \cong S_{j} /\left(K_{j} \cap S_{j}\right)$ is a homomorphic image of $S_{j}$. Choose by (2.11) $q \in \operatorname{Coass}\left(M / K_{j}\right)$. Since Coass $\left(M / K_{j}\right) \subseteq$ Coass $S_{j} \subseteq\left\{p_{j}\right\}$ by (2.12) and the already established equality, we get $p_{j}=q \in$ $\widetilde{\operatorname{Coass}}\left(M / K_{j}\right) \subseteq$ Coass $M$ as desired.

3.2. COROLlary. If $M$ have a secondary representation then Coass $M$ is a finite set. In particular, if $M$ is an Artinian $R$-module then Coass $M$ is a finite set.

3.3. THEOREM. Let $M$ have a secondary representation. Then the following hold

(a) The minimal elements of the set $\mathrm{V}(\mathrm{Ann} M)$ belong to Coass $M$.

(b) $\mathrm{Ass}(R /$ Ann $M) \subseteq$ Coass $M$.

(c) The sets $\mathrm{V}(\operatorname{Ann} M)$, $\tilde{A s s}(R / A n n ~ M)$ and Coass $M$ have the same minimal elements.

Proof. (a) Let $M=S_{1}+S_{2}+\cdots+S_{n}$ be a minimal secondary representation of $M$ where $S_{i}$ is a $p_{i}$-secondary for $i=1,2, \ldots, n$. We have the sequence $\oplus S_{i} \rightarrow M \rightarrow 0$ is exact. Thus $\cap$ Ann $S_{i}=\operatorname{Ann} M$. Let $p$ be a minimal element of the set $\mathrm{V}(\operatorname{Ann} M)$. Then $p$ is a minimal element of the set $\mathrm{V}\left(\right.$ Ann $\left.S_{i}\right)$ for some $1 \leq i \leq n$. Since nil $S_{i}=p_{i}$ we have $p=p_{i}$ and hence $p \in$ Att $S_{i}$. Now the assertion follows from (3.1).

(b) Since Ann $M=\cap \operatorname{Ann} S_{i}$ and Ann $S_{i}$ is a $p_{i}$ primary ideal we have $R /$ Ann $M$ has primary decomposition and A $\widetilde{s s}(R /$ Ann $M) \subseteq$ Att $M$.

(c) We know that $\widetilde{\operatorname{Coass}}_{R} M=\left\{p_{1}, p_{2}, \ldots, p_{n}\right\}$ by (3.1), and that Ann $M \subseteq$ Ann $S_{i}$ for any $1 \leq i \leq n$. Therefore $\mathrm{V}($ Ann $M)$ and Coass $M$ have the same minimal elements by (a). Since Ann $M \subseteq p$ for any $p \in \mathrm{A} \widetilde{\mathrm{ss}}(R / \operatorname{Ann} M)$ the assertion follows from (b).

3.4. TheORem. Let $M$ be a Noetherian R-module. For an injective R-module $E$ we have

Coass $\operatorname{Hom}(M, E)=\{p \in \operatorname{A\widetilde {ss}} M \mid p \subseteq q$ for some $q \in \mathrm{A} \widetilde{\mathrm{ss}} E\}$.

Proof. It follows from [5, Lemma 4] and (3.1).

3.5. Remark. Let $M$ be an $R$-module and let $E$ be an injective $R$-module. Then

$\{p \in \operatorname{Ass} M \mid p \subseteq q$ for some $q \in \operatorname{Ass} E\} \subseteq$ Coass $\operatorname{Hom}(M, E)$, cf. [10, Remark after (1.17)] and (2.10) (a). But equality does not hold in general, cf. [10, Example after (1.8)].

Assume $R$ is a Noetherian ring. In [8] Sharp has found the set of attached 
primes of injective modules $E$ and in [9] Toroghy and Sharp have found the set of attached primes of $\operatorname{Hom}(M, E)$ for any finite module $M$ and injective module $E$. These results are particular cases of the following.

3.6. Theorem. Assume $R$ is a Noetherian ring. Let the zero submodule of $R$-module $M$ have a primary decomposition and let $0=N_{1} \cap N_{2} \cap \cdots \cap N_{n}$ be a minimal primary decomposition of the zero submodule of $M$ where $N_{i}$ is a $p_{i}$ primary submodule of $M$ for $i=1,2, \ldots, n$. For an injective $R$-module $E$,

$\operatorname{Coass} \operatorname{Hom}(M, E)=\{p \in \operatorname{Ass} M \mid p \subseteq q$ for some $q \in \operatorname{Ass} E\}$

Proof. First note that A $\widetilde{s s} M=\left\{p_{1}, p_{2}, \ldots, p_{n}\right\}=A \widetilde{A s} M$ by (1.2) and (1.3). There exists an exact sequence $0 \rightarrow M \rightarrow \oplus M / N_{i}$. Thus the sequence $\oplus \operatorname{Hom}\left(M / N_{i}, E\right) \rightarrow \operatorname{Hom}(M, E) \rightarrow 0$ is exact. Therefore Coass $\operatorname{Hom}(M, E) \subseteq \cup \operatorname{Coass} \operatorname{Hom}\left(M / N_{i}, E\right)$. Since for any $a \in R$ the homothety $M / N_{i} \stackrel{a \cdot}{\longrightarrow} M / N_{i}$ is either injective or nilpotent we have $\operatorname{Hom}\left(M / N_{i}, E\right) \stackrel{a \cdot}{\longrightarrow} \operatorname{Hom}\left(M / N_{i}, E\right)$ is either surjective or nilpotent for $1 \leq i \leq n$. Thus $\operatorname{Hom}\left(M / N_{i}, E\right)$ is either $p_{i}$-secondary or zero. On the other hand if $\operatorname{Hom}\left(M / N_{i}, E\right) \neq 0$ then $\operatorname{Hom}\left(R / p_{i}, E\right) \neq 0$ by (1.8), and hence $p_{i} \subseteq q$ for some $q \in$ Ass $E$ by (1.7). Thus $\operatorname{Coass} \operatorname{Hom}(M, E) \subseteq$ $\{p \in$ Ass $M \mid p \subseteq q$ for some $q \in$ Ass $E\}$. Now the assertion follows from (3.5).

\section{Conilradical.}

In this section we introduce the notion of conilradical (dual notion to that of nilradical).

4.1. Definition. For an $R$-module $M$ we denote the conilradical of $M$ by conil $(M)$ defined as the set of all elements $a \in R$ such that for each cocyclic homomorphic image $L$ of $M$ there exists $n \in \mathrm{N}$ with $a^{n} L=0$.

4.2. THEOREM. For any $R$-module $M$ we have

$$
\operatorname{conil}(M)=\bigcap_{p \in \text { Coass } M} p
$$

Proof. Let $a \in \operatorname{conil}(M)$ and let $p \in$ Coass $M$. It follows that there exists a cocyclic homomorphic image $L$ of $M$ such that $p$ is a minimal element in $\mathrm{V}($ Ann $L)$. Thus there exists $t \in \mathrm{N}$ such that $a^{t} L=0$ by the definition. Since Ann $L \subseteq p$, we have $a^{t} \in p$, and hence $a \in p$.

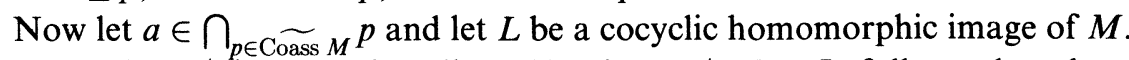
Assume that $a^{t} \notin$ Ann $L$ for all $t \in \mathrm{N}$. Thus $a \notin$ nil $L$. It follows that there exists $q \in \operatorname{Spec}(R)$ such that Ann $L \subseteq q$ and $a \notin q$. Therefore $q \in \operatorname{Cosupp} M$ and $a \notin q$. Now the contradiction follows from (3.7). 
4.3. Corollary. Let $M$ be an $R$-module. Then the following areequivalent

(i) Coass $M$ has exactly one element.

(ii) $\operatorname{conil}(\boldsymbol{M})=\mathrm{w}(\boldsymbol{M})$.

Proof. "(i) $\Rightarrow$ (ii)" It follows from (4.2) and (2.15).

"(ii) $\Rightarrow$ (i)" For any $p \in$ Coass $M$ we have $(M) \subseteq p \subseteq \mathrm{w}(M)$ by (4.2) and (2.15). Now the assertion follows.

\section{Functorial results.}

Let $\mathscr{P}$ be the class of all $R$-modules $M$ such that the zero submodule has primary decomposition (or $M=0$ ), and let $\mathscr{S}$ be the class of all $R$-modules having secondary representation (or being zero). In this section we show some functorial relation between these two classes.

5.1. THEOREM. Let $\mathrm{F}$ be a linear functor over the category of $R$-modules. Then the following hold

(a) If $\mathrm{F}$ is left exact and covariant and if $M \in \mathscr{P}$ then $\mathrm{F}(M) \in \mathscr{P}$. In particular, If $F$ is a flat $R$-module and $M \in \mathscr{P}$ then $M \otimes F \in \mathscr{P}$, and if $M \in \mathscr{P}$ then $\operatorname{Hom}(N, M) \in \mathscr{P}$ for any $R$-module $N$.

(b) If $\mathrm{F}$ is right exact and contravariant and if $M \in \mathscr{P}$ then $\mathrm{F}(M) \in \mathscr{S}$. In particular, If $E$ is an injective $R$-module and $M \in \mathscr{P}$ then $\operatorname{Hom}(M, E) \in \mathscr{S}$.

(c) If $\mathrm{F}$ is right exact and covariant and if $M \in \mathscr{S}$ then $\mathrm{F}(M) \in \mathscr{S}$. In particular, If $M \in \mathscr{S}$ then $M \otimes N \in \mathscr{S}$. In addition, If $P$ is a projective $R$-module and $M \in \mathscr{S}$ then $\operatorname{Hom}(P, M) \in \mathscr{S}$.

(d) If $\mathrm{F}$ is left exact and contravariant and if $M \in \mathscr{S}$ then $\mathrm{F}(M) \in \mathscr{P}$. In particular, If $M \in \mathscr{S}$ then $\operatorname{Hom}(M, N) \in \mathscr{P}$ for any $N$.

Proof. First let $M \in \mathscr{P}$ and let $0=N_{1} \cap N_{2} \cap \cdots \cap N_{n}$ be a minimal primary decomposition of the zero submodule of $M$ where $N_{i}$ is a $p_{i}$-coprimary submodule of $M$ for $i=1,2, \ldots, n$.

(a) Since for any $a \in R$ the homothety $M / N_{i} \stackrel{a \cdot}{\longrightarrow} M / N_{i}$ is either injective or nilpotent we have $\mathrm{F}\left(M / N_{i}\right) \stackrel{a \cdot}{\longrightarrow} \mathrm{F}\left(M / N_{i}\right)$ is either injective or nilpotent for $1 \leq i \leq n$. Thus $\mathrm{F}\left(M / N_{i}\right)$ is either $p_{i}$-coprimary or zero. On the other hand the exact sequence $0 \rightarrow M \rightarrow \oplus_{i}\left(M / N_{i}\right)$ induce the exact sequence $0 \rightarrow \mathrm{F}(M) \rightarrow \oplus_{i} \mathrm{~F}\left(M / N_{i}\right)$.

(b) Since for any $a \in R$ the homothety $M / N_{i} \stackrel{a}{\longrightarrow} M / N_{i}$ is either injective or nilpotent we have $\mathrm{F}\left(M / N_{i}\right) \stackrel{a \cdot}{\longrightarrow} \mathrm{F}\left(M / N_{i}\right)$ is either surjective or nilpotent for $1 \leq i \leq n$. Thus $\mathrm{F}\left(M / N_{i}\right)$ is either $p_{i}$-secondary or zero. On the other hand the exact sequence $0 \rightarrow M \rightarrow \oplus_{i}\left(M / N_{i}\right)$ induce the exact sequence $\oplus_{i} \mathrm{~F}\left(M / N_{i}\right) \rightarrow \mathrm{F}(M) \rightarrow 0$.

Now let $M$ have a secondary representation and $M=S_{1}+S_{2}+\cdots+S_{n}$ is 
a minimal secondary representation of $M$ where $S_{i}$ is a $p_{i}$-secondary submodule of $M$ for $i=1,2, \ldots, n$.

The proof of (c) is similar to the proof (a), and the proof of (d) is similar to the proof (b) using the exact sequence $\oplus S_{i} \rightarrow M \rightarrow 0$.

\section{REFERENCES}

1. N. Bourbaki, Algèbre Commutative, chap. 3 et 4, Herman, Paris 1967.

2. J. Iroz and D. E. Rush, Associated prime ideals in non-Noetherian rings, Canad. J. Math. 36 (1984), 344--360.

3. I. G. Macdonald, Secondary representation of modules over a commutative ring, Sympos. Math. XI (1973), 23--43.

4. H. Matsumura, Commutative ring theory, Cambridge Stud. Adv. Math. 8, 1986.

5. L. Melkersson and P. Schenzel, Asymptotic attached prime ideals related to injective modules, Comm. Algebra 20 (1992), 583--590.

6. L. Melkersson and P. Schenzel, The co-localization of an Artinian module, Proc. Edinburgh Math. Soc., to appear.

7. I. Nishitani, Associated prime ideals of the dual of an Artinian module relative to an injective module, Comm. Algebra 22 (1994), 2651--2667.

8. R. Y. Sharp, Secondary representation for injective modules over commutative Noetherian rings, Proc. Edinburgh Math. Soc. 20 (1976), 143--151.

9. H. A. Toroghy and R. Y. Sharp, Asymptotic behaviour of ideals relative to injective modules over commutative Noetherian rings, Proc. Edinburgh Math. Soc. 34 (1991), 155--160.

10. S. Yassemi, Coassociated primes, Comm. Algebra 23 (1995), 1473--1498.

MATEMATISK INSTITUT

UNIVERSITETSPARKEN 5

DK-2100 KøBENHAVN Ø

DENMARK

E-mail address: yassemi@math.ku.dk
CURRENT ADDRESS:

DEPARTMENT OF MATHEMATICS

UNIVERSITY OF TEHRAN

P.O.BOX 13145-448

TEHRAN-IRAN

E-mail address: yassemi@rose.ipm.ac.ir 